Trascender, Contabilidad y Gestión Núm. 12 (septiembre - diciembre del 2019).

Universidad de Sonora. Departamento de Contabilidad.

ISSN: 2448-6388. Reserva de Derechos 04-2015-04172070800-203.

\title{
Correlación entre colaboración e innovación en el clúster de electrónica y suministros del estado de Aguascalientes
}

\author{
Correlation between collaboration and innovation in the electronics and supplies cluster from the state of \\ Aguascalientes
}

\author{
Andrea Carolina Hernández Santos ${ }^{1}$; Maribel Feria Cruz ${ }^{2}$
}

Recibido: 11 de mayo de 2019.

Aceptado: 19 de agosto de 2019.

DOI: https://doi.org/10.36791/tcg.v11i4.71

JEL: O32. Gestión de la innovación tecnológica.

\section{Resumen}

En un mundo competitivo como el actual, con tanta agilidad y rapidez, acelera la desigualdad en el sector económico. Los grandes cambios producidos en el entorno empresarial y, en especial, el fenómeno de la globalización, han provocado la mejora de la competitividad de las pequeñas y medianas empresas, debido a esto la constitución de un clúster es una medida que ha resultado muy útil para hacerle frente a estos cambios.

La economía mundial, en especial la de los países desarrollados, está dominada por agrupamientos industriales que interactúan muy fuertemente entre sí en aspectos tecnológicos y de conocimientos para generar innovación, y cuyo resultado se manifiesta en crecimiento y generación de valor entre los miembros de éste.
El presente trabajo es un estudio enfocado al clúster de la industria en electrónica del estado de Aguascalientes denominado CELESA A.C., cuyo objetivo general es analizar la relación que existe entre la colaboración y la innovación entre las empresas que conforman el clúster de manera que los esfuerzos se dirijan hacia esas mismas variables.

Esta investigación fue de tipo descriptiva y correlacional con un enfoque cuantitativo. El instrumento fue compuesto por preguntas de opción múltiple y cerradas con medición ordinal en escala de Likert el cual se aplicó a 14 empresas, 7 de ellas pertenecientes al clúster y las otras 7 a empresas que no están afiliadas a él, dado a que la muestra no fue representativa se realizó un estudio de caso para resaltar que en las empresas que sí pertenecen al

\footnotetext{
${ }^{1}$ Andrea Carolina Hernández Santos. Egresada del Instituto Tecnológico de Aguascalientes. Ingeniera en Gestión Empresarial. Correo: carolina.hdz.santos@gmail.com

2 Maribel Feria Cruz. Licenciada en Economía. Doctora en Ciencias Sociales. Docente investigadora del Instituto Tecnológico de Aguascalientes. Correo: fericruz@outlook.com
} 
clúster tengan una relación mayor de colaboración e innovación a diferencia de las que trabajan solo por su cuenta.

El objetivo planteado para el trabajo fue: Analizar la relación que existe entre la colaboración y la innovación entre las empresas que conforman el clúster CELESA.

Una vez analizados los datos recopilados mediante el instrumento propuesto, se encuentra que las dimensiones que engloban las variables bajo estudio arrojan correlaciones significativamente positivas, respecto de la colaboración y la innovación de las empresas que conforman dicho clúster.

Los resultados obtenidos confirman una correlación positiva entre la colaboración y la innovación de las empresas que conforman el clúster de la electrónica, de manera similar sucede para las empresas que se encuentran fuera del clúster, pero en este caso el resultado se alcanza con un menor número de subdimensiones.

Palabras clave: clúster, colaboración, innovación.

\section{Abstract}

In a competitive world, with such agility and speed, inequality in the economic sector is accelerated. The major changes in the business environment and, in particular, the phenomenon of globalization, have led to an improvement in the competitiveness of small and medium-sized enterprises.

The world economy, especially that of developed countries, is dominated by industrial clusters that interact very strongly with each other in technological and knowledge aspects to generate innovation, and whose result is manifested in growth and value generation among the members of the cluster.

The present work is a study focused on the electronics industry cluster in the state of Aguascalientes called CELESA A.C., whose general objective is to analyze the relationship between collaboration and innovation among the companies that make up the cluster so that all efforts are directed towards these same variables.

This research was descriptive and correlated with a quantitative approach. The instrument was composed of multiple choice and closed questions with ordinal measurement in Likert scale which was applied to 14 companies, 7 of them belonging to the cluster and the other 7 to companies that are not affiliated to it, since the sample was not representative a case study was made to highlight that in the companies that do belong to the cluster have a greater relationship of collaboration and innovation as opposed to those that work only on their own.

The objective of the study is: To analyze the relationship between collaboration and innovation among the companies that make up the CELESA cluster.

Once the data collected by means of the proposed instrument has been analyzed, it has been found that the dimensions encompassed by the variables under study show significant positive correlations with respect to collaboration and innovation among the companies making up the cluster.

The results obtained confirm a positive correlation between collaboration and innovation of the companies that make up the electronics cluster, in a similar way it happens for the companies that are outside the cluster, but in this case the result is achieved with a lower number of sub-dimensions.

Keywords: cluster, collaboration, innovation.

\section{Introducción}

El desarrollo económico, la debilidad del peso en los 
mercados extranjeros, la creciente inflación, el mercado globalizado y la aparición de nuevas tecnologías, son algunos desafíos que enfrentan en la actualidad las empresas mexicanas (Berumen y Palacios, 2009, pág. 5; Peres y Garrido, 1998: 26).

La crisis que ocasiona esto, conlleva a buscar nuevas opciones para sobrevivir a los mercados. Una opción favorable, se centra en la creación de grupos industriales conocidos como clústeres, que se crean con la finalidad de que las empresas miembros se vinculen, colaboren, innoven y coordinen, para emprender acciones en conjunto que por sí solas no podrían afrontar, permitiéndoles incrementar su competitividad (Aimacaña y Lara, 2019: 24).

Los clústeres representan una forma novedosa, para abordar la investigación correspondiente con la actividad económica y la formulación de nuevas capacidades de desarrollo en empresas, partiendo de las relaciones comerciales que los miembros de éste establecen, por tal motivo, la colaboración en un clúster desarrolla el intercambio de conocimiento, fomenta la colaboración, la innovación y el avance tecnológico (Becerra, Serna, y Naranjo, 2013: 248).

Un beneficio de pertenecer a un clúster en México y en otras partes del mundo, es la posibilidad de recibir apoyos gubernamentales, para los miembros de un clúster, favoreciendo las ventajas competitivas, permanencia en el mercado, disminución de costos, atracción de nuevos clientes, incremento de productividad, ventajas de integración, entre otras (DiazAriza et al., 2019: 72).

Para alcanzar todos los beneficios de un clúster, se debe promover el desarrollo de una cultura empresarial en la que se pongan en práctica valores y conocimientos, para que la información fluya conjuntamente entre las empresas que lo componen, buscando un "ganar-ganar", situación que puede simplificarse, cuando se tiene un área de desarrollo común (Tomer, Castillo-Navarro, Barcelo-Valenzuela, y León-Duarte, 2009: 37).

En los clústeres especializados las empresas se encuentran enfocadas en áreas comunes. Un clúster especializado, también es una opción para el progreso de la economía, la colaboración y la innovación, ya que, logra el progreso económico, incrementando y aumentando la creación de nexos entre miembros de un mismo sector de desarrollo.(Zayas, Zayas y Heiras, 2017: 533).

Aguascalientes es una ciudad localizada en el centro de México, que ha sido reconocida como el "Pequeño Gigante en Inversiones”, además, ofrece condiciones favorables para el desarrollo de empresas, contemplando esto, el gobierno estatal ha impulsado la formación de clústeres, para mantener la competitividad industrial del estado, actualmente, se han formado diez clústeres industriales y de servicios, enfocados en los sectores estratégicos de la entidad, que incluyen las industrias del mueble, automotriz, tecnologías de la información, textil, electrónica, robótica, exportaciones, transporte, productos municipales y alimentos (Martínez y Feria, 2016: 3).

De acuerdo con Zavala (2014) México es uno de los principales países exportadores y ensambladores del mundo, en él se ubican 9 de las 10 principales empresas transnacionales de servicio de manufactura, por lo que se ha posicionado como un importante destino de inversión” (Zavala, 2014: 27).

En el caso de Aguascalientes el éxito es similar, gracias al crecimiento que se ha registrado en la industria 
manufacturera automotriz, derivado de las inversiones que se han presentado en el estado.

Un sector de especial relevancia en México, en el cual se ha tratado de posicionar al país mediante diferentes esquemas es el electrónico, ya que, se busca tener un éxito similar al que se ha tenido en la industria automotriz, por lo que la generación de clústeres empresariales especializados en electrónica es un enfoque de interés. (León y Rodríguez, 2017: 881)

En la presente investigación, se aborda un estudio de caso comparativo para valorar la relación entre la colaboración y la innovación, entre las empresas que conforman el clúster denominado Consejo de la Electrónica y Suministros del Estado de Aguascalientes (CELESA), y las empresas que se encuentran fuera de este clúster, pero pertenecen al mismo ramo.

Lo anterior, permite identificar la forma en la que las empresas pertenecientes a un clúster tecnológico como CELESA, se organizan y vinculan para alcanzar objetivos, obteniendo mayores niveles de colaboración e innovación. Aunado a esto, se analizan las diferencias entre las empresas que se encuentran adscritas al clúster, en comparación con las que no lo están.

\section{Antecedentes}

Reche, (2019: 3) menciona que los clústeres son grupos geográficamente próximos de compañías interconectadas, vinculadas por características comunes y complementarias, incluyendo empresas de productos finales o servicios, proveedores, instituciones financieras y empresas de industrias conexas.

El surgimiento de los clústeres tiene sus orígenes en 1994 con los trabajos de Michael Porter, donde se destacan las capacidades que tienen las agrupaciones industriales, para potenciar la competitividad, la colaboración e innovación en la industria, por lo que la formación de éstos se ha justificado en producir grupos competentes, que resultan de las transferencias tecnológicas entre empresas, intercambio de mano de obra especializada, reducción de costos logísticos y soporte compartido de proveedores especializados, como lo describe Reche, 2019.

Los clústeres ya se han relacionado previamente, con las variables de colaboración e innovación, como se busca en este trabajo, para el estudio de caso del clúster CELESA. Algunos de los trabajos recientes, que sustentan estas correlaciones entre dichas variables se describen en la tabla 1 . Se presenta su análisis detallado.

Tabla 1. Relación de trabajos que exponen la relación de las variables de colaboración e innovación

\begin{tabular}{|l|l|l|}
\hline Año & Trabajo & Descripción \\
\hline 2019 & $\begin{array}{l}\text { Sistemas regionales de innovación agroalimentarios de } \\
\text { Colombia: un análisis factorial y de clúster para la } \\
\text { industria (Arias y Alarcón, 2019). }\end{array}$ & $\begin{array}{l}\text { Análisis multivariado de los clústeres } \\
\text { agroalimentarios incluyendo la innovación y } \\
\text { competitividad, en Colombia. }\end{array}$ \\
\hline 2019 & $\begin{array}{l}\text { Entretejiendo innovación y colaboración en los } \\
\text { Colectivos de Food Truck de Tijuana (Díaz-Carrión, } \\
\text { Cruz-Estrada, y Páez-Pérez, 2019). }\end{array}$ & $\begin{array}{l}\text { Análisis de innovación y colaboración en } \\
\text { empresas de transporte de alimentos en Tijuana, } \\
\text { México. }\end{array}$ \\
\hline
\end{tabular}


Tabla 1. Continuación...

\begin{tabular}{|c|c|c|}
\hline Año & Trabajo & Descripción \\
\hline 2018 & $\begin{array}{l}\text { Clúster virtual nueva alternativa a la competitividad } \\
\text { eficaz en las empresas (Fernández-Hurtado, Castillo- } \\
\text { Triana, y Martínez-Martínez, 2018). }\end{array}$ & $\begin{array}{l}\text { Planteamiento innovativo de clúster virtual, } \\
\text { Colombia. }\end{array}$ \\
\hline 2018 & $\begin{array}{l}\text { Innovación y competitividad en la industria de } \\
\text { software (Rodríguez-Albor, 2018) }\end{array}$ & $\begin{array}{l}\text { Análisis de diseño de instrumento para medir } \\
\text { Innovación en empresas de software en Jalisco, } \\
\text { México. }\end{array}$ \\
\hline 2016 & $\begin{array}{l}\text { Cooperar para Innovar, El caso de las iniciativas } \\
\text { clúster del sector TI de Sinaloa, México (IIzábal y } \\
\text { Valenzuela, 2016) }\end{array}$ & $\begin{array}{l}\text { Análisis con estadístico de colaboración e } \\
\text { innovación en clúster de Tecnologías de } \\
\text { Información (TIC’s), Sinaloa, México. }\end{array}$ \\
\hline 2016 & $\begin{array}{l}\text { Surgimiento y evolución de clústeres tecnológicos en } \\
\text { regiones de bajo desarrollo: Cuenca, Ecuador (Cuesta- } \\
\text { Delgado, 2016) }\end{array}$ & $\begin{array}{l}\text { Analiza clústeres tecnológicos y realiza una } \\
\text { mención detallada de la colaboración en clústeres } \\
\text { y el efecto en la innovación, en Ecuador. }\end{array}$ \\
\hline 2013 & $\begin{array}{l}\text { Colaboración y competencia como antecedentes de la } \\
\text { innovación de producto. ¿Aplican las empresas nuevas } \\
\text { y establecidas una lógica diferenciada? (Antolín- } \\
\text { López, Martínez-del-Río, y Céspedes-Lorente, 2013) }\end{array}$ & $\begin{array}{l}\text { Artículo que detalla el efecto de la innovación } \\
\text { como resultado de la colaboración entre empresas } \\
\text { en el diseño de un producto, España. }\end{array}$ \\
\hline 2013 & $\begin{array}{l}\text { Redes empresariales locales, investigación y desarrollo } \\
\text { e innovación en la empresa. Clúster de herramientas de } \\
\text { Caldas, Colombia (Becerra et al., 2013) }\end{array}$ & $\begin{array}{l}\text { Se realiza un análisis del estado del arte entorno a } \\
\text { la colaboración y su relación con la innovación } \\
\text { en grupos empresariales, en Colombia. }\end{array}$ \\
\hline 2005 & $\begin{array}{l}\text { Competitividad e innovación: Teoría versus Práctica en } \\
\text { la medida de competitividad turística (Domareski-Ruiz } \\
\text { y Chim-Miki, 2005). }\end{array}$ & $\begin{array}{l}\text { Se describe la colaboración e innovación en el } \\
\text { sector turístico, Brasil. }\end{array}$ \\
\hline
\end{tabular}

Fuente: Elaboración propia. Análisis del estado del arte. 


\section{Planteamiento del Problema}

En México las empresas del ramo electrónico, tienen elementos y componentes para la producción de diversos artículos electrónicos, además, se ha ido impulsando este sector, buscando convertirlo en un área de desarrollo importante del país, como se describe en Zavala, 2014 (pág. 6).

Los cambios que vive la industria de la electrónica se dan con mayor rapidez y de manera indefinida, además de influenciarse por diversas amenazas, todo ello resultado del efecto de globalización y la competencia por la reducción de costos. Las empresas deben adaptarse a estos efectos, creando nuevas estrategias de trabajo. Por lo anterior, se han empezado a establecer lazos de colaboración y los gobiernos han impulsado la clusterización de éstos, con la finalidad de mantener la competencia de la industria electrónica mexicana, que actualmente cubre el $24 \%$ de las exportaciones no petroleras en la nación, de acuerdo con lo indicado en Zavala, (2014: 14).

Es notorio que el impacto que tienen los clústeres en el desarrollo económico del país es fuerte por los factores que están involucrados: investigación y desarrollo, así como por los entes que participan: gobierno, empresa y universidades (Castro-Macías, 2012). Por tal motivo, la clusterización debe llevarse también a la industria electrónica en el estado de Aguascalientes.

En Aguascalientes existen ocho clústeres, incluyendo el clúster de electrónica CELESA, que está conformado por nueve empresas del sector electrónico e institutos de educación superior.

El objetivo general al cual se le busca dar respuesta en este trabajo de investigación es analizar la relación que existe entre la colaboración y la innovación entre las empresas que conforman el clúster CELESA, para que con ello exista evidencia que justifique la formación de clústeres en Aguascalientes, cuando las empresas desean un incremento en su nivel de innovación.

Se pretende que con base en este objetivo, los miembros del clúster conozcan los beneficios de pertenecer a éste, específicamente en las variables de colaboración e innovación, de tal forma que se logre el mejoramiento y/o desarrollo de sus capacidades, con lo que podrán motivar la clusterización de sus empresas.

\section{Alcances y Limitaciones}

Este estudio se realiza con la participación de empresas del clúster CELESA y con empresas del ramo electrónico, pero no pertenecientes a éste, éstas últimas, con la finalidad de contar con un grupo de control, para analizar la relación que hay entre la colaboración y la innovación.

Dado que las empresas del clúster CELESA se localizan únicamente en el estado de Aguascalientes, los resultados que se obtengan solo serán aplicables esta localidad.

La fiabilidad de los resultados de esta investigación, se encuentran ligados al porcentaje de empresas adscritas al clúster CELESA, que decidan participar en la muestra, ya que, la representatividad del grupo se ve afectada con la ausencia de datos de sus miembros registrados.

Derivado del máximo tamaño de muestra para los grupos, este trabajo es únicamente un estudio de caso.

\section{Desarrollo}

El presente estudio es de tipo cuantitativo, transversal, con orientación descriptivo y no experimental y por la manera en la que se analizan las variables es bivariado y correlacional. 
Este estudio es de tipo cuantitativo pues se contabilizarán los resultados obtenidos del instrumento aplicado a empresas que integran el clúster CELESA.

Los diseños de investigación transversal, recolectan datos en un solo momento, en un tiempo único, su propósito es describir variables y analizar su incidencia e interrelación en un momento dado (Hernández-Sampieri et al., 2006: 208).

El propósito de este diseño, es comenzar a conocer una variable o un conjunto de variables, una comunidad, un contexto, un evento o una situación, se trata de una exploración inicial en un momento especifico (Hernández-Sampieri et al., 2006: 208).

Se dice que este estudio es bivariado, ya que, cuenta con dos variables las cuales son la colaboración y la innovación de las empresas que integran el clúster CELESA.

$\mathrm{Al}$ ser un estudio de caso correlacional, se describe a las relaciones entre dos o más categorías, conceptos o variables en un momento determinado, debido a esto la investigación pretende medir el grado de relación de dos variables que son la colaboración y la innovación de las empresas que conforman el clúster CELESA (Hernández-Sampieri et al., 2006: 99).

Este estudio de caso se realizó a partir del segundo semestre del año 2019, se caracteriza por ser transversal, descriptivo realizado a través de un cuestionario estructurado con respuestas cerradas mediante la escala de Likert, nominal y dicotómica; el modelo del instrumento es adoptado de investigadores italianos de la
Región Emilia Romagna, Italia, mismo que corresponde a una metodología cuantitativa.

\section{Población y muestra}

Para efectos de esta investigación, la población de estudio, son las 9 empresas electrónicas integrantes del clúster CELESA, A.C. es importante mencionar que dicho clúster se encuentra agrupado por empresas del sector electrónico, universidades e institutos de investigación.

Dado a que las empresas del clúster representan una población pequeña y además, existe el riesgo de que no todos los miembros de este puedan contestar, se opta por recabar datos de otras empresas externas del clúster, pero afines al mismo sector, estas empresas son en su totalidad independientes una de la otra.

Por lo anterior, en esta investigación recae en un estudio de caso, puesto que de determinará la relación que existe entre la colaboración y la innovación dentro y fuera del clúster.

Se optó por delimitar la muestra a 14 empresas, la cual se conforma de siete empresas pertenecientes al clúster y las otra siete por empresas externas a éste.

\section{Operacionalización de variables}

Las variables consideradas en este estudio de las cuales se realiza su operacionalización son:

- Colaboración

- Innovación 
En las siguientes tablas se describen sus dimensiones y sus subdimensiones

Tabla 2. Dimensión y subdimensión de la variable colaboración

\section{Variable: Colaboración}

“La relación individual establecida por una empresa con otra, interactuando en un acuerdo para el trabajo común con la finalidad de generar sinergias y comprometiéndose a la realización de un proceso de generación de valor, mismo que se comparte” (Ojeda Goméz, 2009: 46).

\begin{tabular}{|c|c|}
\hline Dimensión & Subdimensión \\
\hline Actividades de colaboración & Colaborar para producir \\
\hline Actividades de colaboración & Colaborar para Innovar \\
\hline Actividades de colaboración & Colaborar para el Mercado \\
\hline Actividades de colaboración & Colaborar para Administrar \\
\hline
\end{tabular}

Fuente: Elaboración propia.

Tabla 3. Dimensión y subdimensión de la variable innovación

\begin{tabular}{|c|c|}
\hline \multicolumn{2}{|c|}{ Variable: Innovación } \\
“La introducción de nuevos bienes y servicios, adaptándose a los cambios de la demanda de los consumidores y a los \\
nuevos competidores” (Bateman y Snell, 2009: 12).
\end{tabular}

Fuente: Elaboración propia. 


\section{Resultados}

Posterior a la construcción del instrumento de valoración para correlacionar colaboración e innovación, éste se aplica a las empresas del clúster y a los grupos de control, una vez hecho esto, se analiza su nivel de confiabilidad y de correlación para las variables antes mencionadas.

\section{Confiabilidad}

La confiabilidad de un instrumento de medición se refiere al grado en que su aplicación repetida al mismo sujeto u objeto produce resultados iguales” Hernández Sampieri et al. (2006: 277). Pardo y Ruiz, (2002: 590) definen la confiabilidad como "la capacidad de la escala para medir de forma consistente precisa y sin error la característica que se desea medir”, en forma similar, Castañeda Jiménez (2011: 182) la define como "la capacidad de un instrumento de arrojar resultados equivalentes entre los respondedores, independientemente de quién lo aplique”.

Se utilizan diversos índices en la literatura para cuantificar la confiabilidad, pero en general, en estas técnicas se tienen coeficientes en rangos entre cero y uno para valorar la confiabilidad, teniendo nula confiabilidad para un coeficiente con valor de cero y confiabilidad plena para un coeficiente con valor de uno (Castro Macías, 2012: 141). Sin embargo, de acuerdo con Hernández Sampieri et al. (2006: 288) no existe una regla que indique que valor aceptar como confiable, pero en forma general se puede aceptar su mapeo por niveles, teniendo menos de 0.5 para un nivel de baja confiablidad, 0.5 a 0.75 para una confiabilidad media, de 0.75 a 0.90 para una confiabilidad aceptable y si es mayor a 0.90 se tiene una alta confiablidad.

Para el análisis de confiabilidad con alfa de Cronbach en esta investigación, se evalúan cada una de las dimensiones y subdimensiones del instrumento. Los resultados de confiabilidad para empresas del clúster se muestran en la tabla 4 para las dimensiones y en la tabla 5 para las subdimensiones. Los resultados de confiabilidad para empresas ajenas al clúster se muestran en la tabla 6 para las dimensiones y en la tabla 7 para las subdimensiones.

Tabla 4. Análisis de confiabilidad de Cronbach para las dimensiones en empresas del clúster

\begin{tabular}{|c|c|}
\hline \multicolumn{2}{|c|}{ Estadísticas de fiabilidad en análisis con empresas del clúster entre dimensiones } \\
\hline Alfa de Cronbach & N de elementos \\
\hline 0.925 & 2 \\
\hline
\end{tabular}

Fuente: Elaboración propia. 
Tabla 5. Análisis de confiabilidad de Cronbach para las subdimensiones en empresas del clúster

\begin{tabular}{|c|c|c|c|}
\hline \multicolumn{3}{|c|}{ Estadísticas de fiabilidad con empresas del clúster entre subdimensiones } \\
\hline \multirow{3}{*}{ Dimensiones } & Subdimensiones & Alfa de Cronbach & N de elementos \\
\hline \multirow{3}{*}{ Actividades de Colaboración } & Colaborar para Producir & 0.956 & 8 \\
\cline { 2 - 4 } & Colaborar para Innovar & 0.955 & 8 \\
\cline { 2 - 4 } & Colaborar para Mercado & 0.947 & 8 \\
\cline { 2 - 4 } & Colaborar para Administrar & 0.959 & 8 \\
\hline \multirow{3}{*}{ Actividades de Innovación } & Tipos de Innovación & 0.977 & 8 \\
\cline { 2 - 4 } & Rubros de Innovación & 0.978 & 8 \\
\cline { 2 - 4 } & Capacitación de Innovación & 0.978 & 8 \\
\cline { 2 - 4 } & Financiamiento de Innovación & 0.992 & 8 \\
\hline
\end{tabular}

Fuente: Elaboración propia.

Tabla 6. Análisis de confiabilidad de Cronbach para las dimensiones en empresas ajenas al clúster

\begin{tabular}{|c|c|}
\hline \multicolumn{2}{|c|}{$\begin{array}{c}\text { Estadísticas de fiabilidad en análisis con empresas ajenas al } \\
\text { clúster entre dimensiones }\end{array}$} \\
\hline Alfa de Cronbach & N de elementos \\
\hline 0.856 & 2 \\
\hline
\end{tabular}

Fuente: Elaboración propia. 
Tabla 7. Análisis de confiabilidad de Cronbach para las subdimensiones en empresas ajenas al clúster

\begin{tabular}{|c|c|c|c|}
\hline \multicolumn{4}{|c|}{ Estadísticas de fiabilidad con empresas ajenas al clúster entre subdimensiones } \\
\hline \multirow{2}{*}{ Dimensiones } & Subdimensiones & Alfa de Cronbach & N de elementos \\
\hline \multirow{3}{*}{ Actividades de Colaboración } & Colaborar para Producir & 0.839 & 5 \\
\cline { 2 - 4 } & Colaborar para Innovar & 0.852 & 5 \\
\cline { 2 - 4 } & Colaborar para Mercado & 0.846 & 5 \\
\cline { 2 - 4 } & Colaborar para Administrar & 0.863 & 5 \\
\hline Actividades de Innovación & Agentes de Innovación & 0.975 & 5 \\
\hline
\end{tabular}

Fuente: Elaboración propia.

\section{Correlación}

De acuerdo con lo indicado por Castro Macías (2012, pág. 143), los coeficientes de rho de Spearman, "son medidas de correlaciones para variables en un nivel de medición ordinal, los individuos u objetos de la muestra pueden ordenarse por rangos jerarquizados” (Hernández Sampieri et al., 2006, pág. 480). De acuerdo con Briones (2003, pág. 204) y Stevenson (2005, pág. 457) la fórmula para calcular el coeficiente de correlación $\rho$ de Spearman es la indicada en la ecuación (1).

$$
\rho=1-\frac{6 \sum D^{2}}{n\left(n^{2}-1\right)}
$$

Donde:

$\sum D^{2}{ }_{i}=$ suma de los cuadrados de las diferencias entre rangos y $n:=$ número de clases u observaciones.
El valor de $\rho$ expresa el nivel de correlación con -1.0 para una correlación negativa perfecta, +1.0 para una correlación positiva perfecta y 0 para una correlación nula (Castro Macías, 2012: 144). Aunado a esto, los niveles de correlación acuerdo con Akoglu (2018: 42) pueden establecerse con base en la magnitud de $\rho$, con una Moderada Correlación (MC) para valores de 0.4 a 0.6, Buena Correlación (BC) para valores de 0.6 a 0.8 y Alta Correlación (AC) con valores de 0.8 a 1.0 .

Además, para validar el nivel de correlación en la hipótesis propuesto por $\rho$ debe considerarse la significatividad que puede ser aceptada al 0.01 o 1\% de error, entregando una alta confianza en la hipótesis, o al 0.05 o $5 \%$ de error con un nivel de significatividad aceptable (Castro Macías, 2012: 145).

El análisis de correlación entre las dimensiones y subdimensiones significativas para las empresas encuestadas que se encuentran en el clúster se muestran las tablas siguientes: 
La tabla 8 muestra el análisis de correlación de Spearman entre las dimensiones de este trabajo, que incluyen a las actividades de colaboración e innovación, contemplando a los 7 encuestados de las empresas del clúster. Como resultado se alcanza una alta significancia menor al 0.01 y una alta correlación de 1.000 .

Tabla 8. Análisis de correlación de Spearman para las dimensiones en empresas del clúster

\begin{tabular}{|c|c|c|c|c|}
\hline \multicolumn{5}{|c|}{ Correlaciones en empresas del clúster entre dimensiones } \\
\hline & & & $\begin{array}{l}\text { Actividades de } \\
\text { Colaboración }\end{array}$ & $\begin{array}{l}\text { Actividades de } \\
\text { Innovación }\end{array}$ \\
\hline \multirow{6}{*}{$\begin{array}{c}\text { Rho de } \\
\text { Spearman }\end{array}$} & \multirow{3}{*}{$\begin{array}{l}\text { Actividades de } \\
\text { Colaboración }\end{array}$} & Coeficiente de correlación & 1.000 & $1.000^{* *}$ \\
\hline & & Sig. (bilateral) & & \\
\hline & & $\mathrm{N}$ & 7 & 7 \\
\hline & \multirow{3}{*}{$\begin{array}{l}\text { Actividades de } \\
\text { Innovación }\end{array}$} & Coeficiente de correlación & $1.000^{* *}$ & 1.000 \\
\hline & & Sig. (bilateral) & & \\
\hline & & $\mathrm{N}$ & 7 & 7 \\
\hline
\end{tabular}

Fuente: Elaboración propia.

Las tablas siguientes, se muestran el análisis de correlación de Spearman entre la subdimensión de colaboración colaborar para producir y las subdimensiones de innovación, que incluyen tipos de innovación, rubros de innovación, capacitación de innovación y financiamiento de innovación. Como resultado contemplando a las empresas del clúster, se alcanza una alta significancia menor al 0.01 , para cada uno de los análisis y una alta correlación desde 0.900 hasta 1.000 . 
Tabla 9. Análisis de correlación de Spearman para las subdimensiones Colaboración para Producir y Tipo de Innovación en empresas del clúster

\begin{tabular}{|c|c|c|c|c|}
\hline \multicolumn{5}{|c|}{ Correlaciones en empresas del clúster entre subdimensiones } \\
\hline & & & $\begin{array}{l}\text { Colaborar para } \\
\text { Producir }\end{array}$ & Tipo de Innovación \\
\hline \multirow{6}{*}{$\begin{array}{c}\text { Rho de } \\
\text { Spearman }\end{array}$} & \multirow{3}{*}{$\begin{array}{l}\text { Colaborar para } \\
\text { Producir }\end{array}$} & Coeficiente de correlación & 1.000 & $.900^{* *}$ \\
\hline & & Sig. (bilateral) & & 0.006 \\
\hline & & $\mathrm{N}$ & 7 & 7 \\
\hline & \multirow{3}{*}{ Tipo de Innovación } & Coeficiente de correlación & $.900^{* *}$ & 1.000 \\
\hline & & Sig. (bilateral) & 0.006 & \\
\hline & & $\mathrm{N}$ & 7 & 7 \\
\hline
\end{tabular}

Fuente: Elaboración propia.

Tabla 10. Análisis de correlación de Spearman para las subdimensiones Colaboración para Producir y Rubros de Innovación en empresas del clúster

\begin{tabular}{|c|c|c|c|c|}
\hline \multicolumn{5}{|c|}{ Correlaciones en empresas del clúster entre subdimensiones } \\
\hline \multirow{2}{|c|}{} & $\begin{array}{c}\text { Colabora para } \\
\text { Producir }\end{array}$ & $\begin{array}{c}\text { Rubros de } \\
\text { Innovación }\end{array}$ \\
\hline \multirow{2}{*}{ Rho de Spearman } & $\begin{array}{c}\text { Colabora para } \\
\text { Producir }\end{array}$ & Sig. (bilateral) & 1.000 & $1.000^{* *}$ \\
\cline { 3 - 5 } & \multirow{2}{*}{\begin{tabular}{c} 
Coeficiente de correlación \\
\cline { 3 - 5 }
\end{tabular}} & $\mathrm{N}$ & 7 & 7 \\
\hline
\end{tabular}


Tabla 10. Continuación...

\begin{tabular}{|c|c|c|c|c|}
\hline \multicolumn{5}{|c|}{ Correlaciones en empresas del clúster entre subdimensiones } \\
\hline & & & $\begin{array}{c}\text { Colabora para } \\
\text { Producir }\end{array}$ & $\begin{array}{l}\text { Rubros de } \\
\text { Innovación }\end{array}$ \\
\hline \multirow{3}{*}{ Rho de Spearman } & \multirow{3}{*}{$\begin{array}{l}\text { Rubros de } \\
\text { Innovación }\end{array}$} & Coeficiente de correlación & $1.000^{* *}$ & 1.000 \\
\hline & & Sig. (bilateral) & & \\
\hline & & $\mathrm{N}$ & 7 & 7 \\
\hline \multicolumn{5}{|c|}{ **. La correlación es significativa en el nivel 0,01 (bilateral). } \\
\hline
\end{tabular}

Fuente: Elaboración propia.

Tabla 11. Análisis de correlación de Spearman para las subdimensiones Colaboración para Producir y Capacitación de Innovación en empresas del clúster

\begin{tabular}{|c|c|c|c|c|}
\hline \multicolumn{5}{|c|}{ Correlaciones en empresas del clúster entre subdimensiones } \\
\hline & & & $\begin{array}{c}\text { Colabora para } \\
\text { Producir }\end{array}$ & $\begin{array}{l}\text { Capacitación de } \\
\text { Innovación }\end{array}$ \\
\hline \multirow{6}{*}{$\begin{array}{c}\text { Rho de } \\
\text { Spearman }\end{array}$} & \multirow{3}{*}{$\begin{array}{c}\text { Colabora para } \\
\text { Producir }\end{array}$} & Coeficiente de correlación & 1.000 & $1.000^{* *}$ \\
\hline & & Sig. (bilateral) & & \\
\hline & & $\mathrm{N}$ & 7 & 7 \\
\hline & \multirow{3}{*}{$\begin{array}{l}\text { Capacitación de } \\
\text { Innovación }\end{array}$} & Coeficiente de correlación & $1.000^{* *}$ & 1.000 \\
\hline & & Sig. (bilateral) & & \\
\hline & & $\mathrm{N}$ & 7 & 7 \\
\hline \multicolumn{5}{|c|}{ **. La correlación es significativa en el nivel 0,01 (bilateral). } \\
\hline
\end{tabular}

Fuente: Elaboración propia. 
Tabla 12. Análisis de correlación de Spearman para las subdimensiones Colaboración para Producir y Financiamiento de Innovación en empresas del clúster

\begin{tabular}{|c|c|c|c|c|}
\hline \multicolumn{5}{|c|}{ Correlaciones en empresas del clúster entre subdimensiones } \\
\hline & & & $\begin{array}{l}\text { Colabora para } \\
\text { Producir }\end{array}$ & $\begin{array}{l}\text { Financiamiento de } \\
\text { Innovación }\end{array}$ \\
\hline \multirow{6}{*}{$\begin{array}{c}\text { Rho de } \\
\text { Spearman }\end{array}$} & \multirow{3}{*}{$\begin{array}{l}\text { Colabora para } \\
\text { Producir }\end{array}$} & Coeficiente de correlación & 1.000 & $1.000^{* *}$ \\
\hline & & Sig. (bilateral) & & \\
\hline & & $\mathrm{N}$ & 7 & 7 \\
\hline & \multirow{3}{*}{$\begin{array}{l}\text { Financiamiento de } \\
\text { Innovación }\end{array}$} & Coeficiente de correlación & $1.000^{* *}$ & 1.000 \\
\hline & & Sig. (bilateral) & & \\
\hline & & $\mathrm{N}$ & 7 & 7 \\
\hline
\end{tabular}

Fuente: Elaboración propia.

Las tablas siguientes muestran el análisis de correlación de Spearman entre la subdimensión de colaboración colaborar para innovar y las subdimensiones de innovación, que incluyen tipos de innovación, rubros de innovación, capacitación de innovación y financiamiento de innovación. Como resultado contemplando a las empresas del clúster, se alcanza una alta significancia menor al 0.01, para cada una de los análisis y una alta correlación desde 0.900 hasta 1.000 . 
Tabla 13. Análisis de correlación de Spearman para las subdimensiones Colaboración para Innovar y Tipo de Innovación en empresas del clúster

\begin{tabular}{|c|c|c|c|c|}
\hline \multicolumn{5}{|c|}{ Correlaciones en empresas del clúster entre subdimensiones } \\
\hline & & & $\begin{array}{l}\text { Colaborar para } \\
\text { Innovar }\end{array}$ & Tipo de Innovación \\
\hline \multirow{6}{*}{ Rho de Spearman } & \multirow{3}{*}{$\begin{array}{l}\text { Colaborar para } \\
\text { Innovar }\end{array}$} & Coeficiente de correlación & 1.000 & $.900 * *$ \\
\hline & & Sig. (bilateral) & & 0.006 \\
\hline & & $\mathrm{N}$ & 7 & 7 \\
\hline & \multirow{3}{*}{$\begin{array}{c}\text { Tipo de } \\
\text { Innovación }\end{array}$} & Coeficiente de correlación & $.900 * *$ & 1.000 \\
\hline & & Sig. (bilateral) & 0.006 & \\
\hline & & $\mathrm{N}$ & 7 & 7 \\
\hline
\end{tabular}

Fuente: Elaboración propia.

Tabla 14. Análisis de correlación de Spearman para las subdimensiones Colaboración para Innovar y Rubros de Innovación en empresas del clúster

\begin{tabular}{|c|c|c|c|c|}
\hline \multicolumn{5}{|c|}{ Correlaciones en empresas del clúster entre subdimensiones } \\
\hline & & & $\begin{array}{l}\text { Colaborar para } \\
\text { Innovar }\end{array}$ & $\begin{array}{l}\text { Rubros de } \\
\text { Innovación }\end{array}$ \\
\hline \multirow{3}{*}{ Rho de Spearman } & \multirow{3}{*}{$\begin{array}{l}\text { Colaborar para } \\
\text { Innovar }\end{array}$} & Coeficiente de correlación & 1.000 & $1.000^{* *}$ \\
\hline & & Sig. (bilateral) & & \\
\hline & & $\mathrm{N}$ & 7 & 7 \\
\hline
\end{tabular}


Tabla 14. Continuación...

\begin{tabular}{|c|c|c|c|c|}
\hline \multicolumn{7}{|c|}{ Correlaciones en empresas del clúster entre subdimensiones } \\
\hline \multirow{2}{*}{ Rho de Spearman } & $\begin{array}{c}\text { Colaborar para } \\
\text { Innovar } \\
\text { Rubros de }\end{array}$ & $\begin{array}{c}\text { Rubros de } \\
\text { Innovación }\end{array}$ \\
\cline { 3 - 5 } & \multirow{2}{*}{\begin{tabular}{c} 
Innovación \\
\cline { 3 - 5 }
\end{tabular}} & Sig. (bilateral) & $1.000^{* *}$ & 1.000 \\
\cline { 3 - 5 } & \multirow{2}{*}{$* *$ La correlación es significativa en el nivel 0,01 (bilateral). } & 7 \\
\hline
\end{tabular}

Fuente: Elaboración propia.

Tabla 15. Análisis de correlación de Spearman para las subdimensiones Colaboración para Innovar y Capacitación de Innovación en empresas del clúster

\begin{tabular}{|c|c|c|c|c|}
\hline \multicolumn{5}{|c|}{ Correlaciones en empresas del clúster entre subdimensiones } \\
\hline & & & $\begin{array}{c}\text { Colaborar para } \\
\text { Innovar }\end{array}$ & $\begin{array}{l}\text { Capacitación de } \\
\text { Innovación }\end{array}$ \\
\hline \multirow{6}{*}{$\begin{array}{c}\text { Rho de } \\
\text { Spearman }\end{array}$} & \multirow{3}{*}{$\begin{array}{l}\text { Colaborar para } \\
\text { Innovar }\end{array}$} & Coeficiente de correlación & 1.000 & $1.000^{* *}$ \\
\hline & & Sig. (bilateral) & & \\
\hline & & $\mathrm{N}$ & 7 & 7 \\
\hline & \multirow{3}{*}{$\begin{array}{l}\text { Capacitación de } \\
\text { Innovación }\end{array}$} & Coeficiente de correlación & $1.000^{* *}$ & 1.000 \\
\hline & & Sig. (bilateral) & & \\
\hline & & $\mathrm{N}$ & 7 & 7 \\
\hline
\end{tabular}

Fuente: Elaboración propia. 
Tabla 16. Análisis de correlación de Spearman para las subdimensiones Colaboración para Innovar y Financiamiento de Innovación en empresas del clúster

\begin{tabular}{|c|c|c|c|c|}
\hline \multicolumn{5}{|c|}{ Correlaciones en empresas del clúster entre subdimensiones } \\
\hline & & & $\begin{array}{c}\text { Colaborar para } \\
\text { Innovar }\end{array}$ & $\begin{array}{l}\text { Financiamiento de } \\
\text { Innovación }\end{array}$ \\
\hline \multirow{6}{*}{$\begin{array}{c}\text { Rho de } \\
\text { Spearman }\end{array}$} & \multirow{3}{*}{$\begin{array}{l}\text { Colaborar para } \\
\text { Innovar }\end{array}$} & Coeficiente de correlación & 1.000 & $1.000^{* *}$ \\
\hline & & Sig. (bilateral) & & \\
\hline & & $\mathrm{N}$ & 7 & 7 \\
\hline & \multirow{3}{*}{$\begin{array}{l}\text { Financiamiento de } \\
\text { Innovación }\end{array}$} & Coeficiente de correlación & $1.000^{* *}$ & 1.000 \\
\hline & & Sig. (bilateral) & & \\
\hline & & $\mathrm{N}$ & 7 & 7 \\
\hline \multicolumn{5}{|c|}{ **. La correlación es significativa en el nivel 0,01 (bilateral). } \\
\hline
\end{tabular}

Fuente: Elaboración propia.

Las tablas siguientes muestran el análisis de correlación de Spearman entre la subdimensión de colaboración colaborar para mercado y las subdimensiones de innovación, que incluyen tipos de innovación, rubros de innovación, capacitación de innovación y financiamiento de innovación. Como resultado contemplando a las empresas del clúster, se alcanza una alta significancia menor al 0.01, para cada una de los análisis y una alta correlación desde 0.900 hasta 1.000 . 
Tabla 17. Análisis de correlación de Spearman para las subdimensiones Colaboración para Mercado y Tipo de Innovación en empresas del clúster

\begin{tabular}{|c|c|c|c|c|}
\hline \multicolumn{5}{|c|}{ Correlaciones en empresas del clúster entre subdimensiones } \\
\hline & & & $\begin{array}{l}\text { Colaborar para } \\
\text { Mercado }\end{array}$ & Tipo de Innovación \\
\hline \multirow{6}{*}{ Rho de Spearman } & \multirow{3}{*}{$\begin{array}{c}\text { Colaborar para } \\
\text { Mercado }\end{array}$} & Coeficiente de correlación & 1.000 & $.900 * *$ \\
\hline & & Sig. (bilateral) & & 0.006 \\
\hline & & $\mathrm{N}$ & 7 & 7 \\
\hline & \multirow{3}{*}{$\begin{array}{l}\text { Tipo de } \\
\text { Innovación }\end{array}$} & Coeficiente de correlación & $.900 * *$ & 1.000 \\
\hline & & Sig. (bilateral) & 0.006 & \\
\hline & & $\mathrm{N}$ & 7 & 7 \\
\hline & $* *$. La & ción es significativa en el $\mathrm{l}$ & 01 (bilateral). & \\
\hline
\end{tabular}

Fuente: Elaboración propia.

Tabla 18. Análisis de correlación de Spearman para las subdimensiones Colaboración para Mercado y Rubros de Innovación en empresas del clúster

\begin{tabular}{|c|c|c|c|c|}
\hline \multicolumn{7}{|c|}{ Correlaciones en empresas del clúster entre subdimensiones } \\
\hline \multicolumn{2}{|c|}{} & $\begin{array}{c}\text { Colaborar para } \\
\text { Mercado }\end{array}$ & $\begin{array}{c}\text { Rubros de } \\
\text { Innovación }\end{array}$ \\
\hline \multirow{2}{*}{$\begin{array}{c}\text { Rho de } \\
\text { Spearman }\end{array}$} & $\begin{array}{c}\text { Colaborar para } \\
\text { Mercado }\end{array}$ & Sig. (bilateral) & 1.000 & $1.000^{* *}$ \\
\cline { 3 - 5 } & \multirow{2}{*}{\begin{tabular}{c} 
Coeficiente de correlación \\
\cline { 3 - 5 }
\end{tabular}} & $\mathrm{N}$ & 7 & 7 \\
\hline
\end{tabular}


Tabla 18. Continuación...

\begin{tabular}{|c|c|c|c|c|}
\hline \multicolumn{7}{|c|}{ Correlaciones en empresas del clúster entre subdimensiones } \\
\hline \multirow{2}{*}{$\begin{array}{c}\text { Rho de } \\
\text { Spearman }\end{array}$} & $\begin{array}{c}\text { Colaborar para } \\
\text { Mercado }\end{array}$ & $\begin{array}{c}\text { Rubros de } \\
\text { Innovación }\end{array}$ \\
\hline \multirow{2}{*}{\begin{tabular}{c} 
Innovación \\
\cline { 3 - 4 }
\end{tabular}} & Coeficiente de correlación & $1.000^{* *}$ & 1.000 \\
\cline { 3 - 5 } & \multirow{2}{*}{$* *$ La correlación es significativa en el nivel 0,01 (bilateral). } & 7 \\
\hline
\end{tabular}

Fuente: Elaboración propia.

Tabla 19. Análisis de correlación de Spearman para las subdimensiones Colaboración para Mercado y Capacitación de Innovación en empresas del clúster

\begin{tabular}{|c|c|c|c|c|}
\hline \multicolumn{5}{|c|}{ Correlaciones en empresas del clúster entre subdimensiones } \\
\hline & & & $\begin{array}{c}\text { Colaborar para } \\
\text { Mercado }\end{array}$ & $\begin{array}{l}\text { Capacitación de } \\
\text { Innovación }\end{array}$ \\
\hline \multirow{6}{*}{$\begin{array}{c}\text { Rho de } \\
\text { Spearman }\end{array}$} & \multirow{3}{*}{$\begin{array}{c}\text { Colaborar para } \\
\text { Mercado }\end{array}$} & Coeficiente de correlación & 1.000 & $1.000 * *$ \\
\hline & & Sig. (bilateral) & & \\
\hline & & $\mathrm{N}$ & 7 & 7 \\
\hline & \multirow{3}{*}{$\begin{array}{l}\text { Capacitación de } \\
\text { Innovación }\end{array}$} & Coeficiente de correlación & $1.000 * *$ & 1.000 \\
\hline & & Sig. (bilateral) & & \\
\hline & & $\mathrm{N}$ & 7 & 7 \\
\hline \multicolumn{5}{|c|}{ **. La correlación es significativa en el nivel 0,01 (bilateral). } \\
\hline
\end{tabular}

Fuente: Elaboración propia. 
Tabla 20. Análisis de correlación de Spearman para las subdimensiones Colaboración para Mercado y Financiamiento de Innovación en empresas del clúster

\begin{tabular}{|c|c|c|c|c|}
\hline \multicolumn{5}{|c|}{ Correlaciones en empresas del clúster entre subdimensiones } \\
\hline & & & $\begin{array}{c}\text { Colaborar para } \\
\text { Mercado }\end{array}$ & $\begin{array}{l}\text { Financiamiento de } \\
\text { Innovación }\end{array}$ \\
\hline \multirow{6}{*}{$\begin{array}{c}\text { Rho de } \\
\text { Spearman }\end{array}$} & \multirow{3}{*}{$\begin{array}{l}\text { Colaborar para } \\
\text { Mercado }\end{array}$} & Coeficiente de correlación & 1.000 & $1.000 * *$ \\
\hline & & Sig. (bilateral) & & \\
\hline & & $\mathrm{N}$ & 7 & 7 \\
\hline & \multirow{3}{*}{$\begin{array}{l}\text { Financiamiento de } \\
\text { Innovación }\end{array}$} & Coeficiente de correlación & $1.000 * *$ & 1.000 \\
\hline & & Sig. (bilateral) & & \\
\hline & & $\mathrm{N}$ & 7 & 7 \\
\hline
\end{tabular}

Fuente: Elaboración propia.

Las tablas siguientes muestran el análisis de correlación de Spearman entre la subdimensión de colaboración colaborar para administrar y las subdimensiones de innovación, que incluyen tipos de innovación, rubros de innovación, capacitación de innovación y financiamiento de innovación. Como resultado contemplando a las empresas del clúster, se alcanza una alta significancia menor al 0.01, para cada una de los análisis y una alta correlación desde 0.900 hasta 1.000 . 
Tabla 21. Análisis de correlación de Spearman para las subdimensiones Colaboración para Administrar y Tipo de Innovación en empresas del clúster

\begin{tabular}{|c|c|c|c|c|}
\hline \multicolumn{5}{|c|}{ Correlaciones en empresas del clúster entre subdimensiones } \\
\hline & & & $\begin{array}{c}\text { Colaborar para } \\
\text { Administrar }\end{array}$ & $\begin{array}{c}\text { Tipo de } \\
\text { Innovación }\end{array}$ \\
\hline \multirow{6}{*}{$\begin{array}{c}\text { Rho de } \\
\text { Spearman }\end{array}$} & \multirow{3}{*}{$\begin{array}{l}\text { Colaborar para } \\
\text { Administrar }\end{array}$} & $\begin{array}{c}\text { Coeficiente de } \\
\text { correlación }\end{array}$ & 1.000 & $.900 * *$ \\
\hline & & Sig. (bilateral) & & 0.006 \\
\hline & & $\mathrm{N}$ & 7 & 7 \\
\hline & \multirow{3}{*}{ Tipo de Innovación } & $\begin{array}{c}\text { Coeficiente de } \\
\text { correlación }\end{array}$ & $.900 * *$ & 1.000 \\
\hline & & Sig. (bilateral) & 0.006 & \\
\hline & & $\mathrm{N}$ & 7 & 7 \\
\hline \multicolumn{5}{|c|}{ **. La correlación es significativa en el nivel 0,01 (bilateral). } \\
\hline
\end{tabular}

Fuente: Elaboración propia.

Tabla 22. Análisis de correlación de Spearman para las subdimensiones Colaboración para Administrar y Rubros de Innovación en empresas del clúster

\begin{tabular}{|c|c|c|c|c|}
\hline \multicolumn{5}{|c|}{ Correlaciones en empresas del clúster entre subdimensiones } \\
\hline & & & $\begin{array}{c}\text { Colaborar para } \\
\text { Administrar }\end{array}$ & $\begin{array}{l}\text { Rubros de } \\
\text { Innovación }\end{array}$ \\
\hline \multirow{3}{*}{$\begin{array}{c}\text { Rho de } \\
\text { Spearman }\end{array}$} & \multirow{3}{*}{$\begin{array}{c}\text { Colaborar para } \\
\text { Administrar }\end{array}$} & $\begin{array}{c}\text { Coeficiente de } \\
\text { correlación }\end{array}$ & 1.000 & $1.000^{* *}$ \\
\hline & & Sig. (bilateral) & & \\
\hline & & $\mathrm{N}$ & 7 & 7 \\
\hline
\end{tabular}


Tabla 22. Continuación...

\begin{tabular}{|c|c|c|c|c|}
\hline \multicolumn{7}{|c|}{ Correlaciones en empresas del clúster entre subdimensiones } \\
\hline \multirow{2}{|c|}{} & $\begin{array}{c}\text { Colaborar para } \\
\text { Administrar }\end{array}$ & $\begin{array}{c}\text { Rubros de } \\
\text { Innovación }\end{array}$ \\
\hline \multirow{2}{*}{$\begin{array}{c}\text { Rho de } \\
\text { Spearman }\end{array}$} & $\begin{array}{c}\text { Rubros de } \\
\text { Innovación }\end{array}$ & Sig. (bilateral) & $1.000^{* *}$ & 1.000 \\
\cline { 3 - 5 } & & N & 7 & 7 \\
\hline
\end{tabular}

Fuente: Elaboración propia.

Tabla 23. Análisis de correlación de Spearman para las subdimensiones Colaboración para Administrar y Capacitación de Innovación en empresas del clúster

\begin{tabular}{|c|c|c|c|c|}
\hline \multicolumn{5}{|c|}{ Correlaciones en empresas del clúster entre subdimensiones } \\
\hline & & & $\begin{array}{c}\text { Colaborar para } \\
\text { Administrar }\end{array}$ & $\begin{array}{l}\text { Capacitación de } \\
\text { Innovación }\end{array}$ \\
\hline \multirow{6}{*}{$\begin{array}{c}\text { Rho de } \\
\text { Spearman }\end{array}$} & \multirow{3}{*}{$\begin{array}{c}\text { Colaborar para } \\
\text { Administrar }\end{array}$} & $\begin{array}{c}\text { Coeficiente de } \\
\text { correlación }\end{array}$ & 1.000 & $1.000 * *$ \\
\hline & & Sig. (bilateral) & & \\
\hline & & $\mathrm{N}$ & 7 & 7 \\
\hline & \multirow{3}{*}{$\begin{array}{l}\text { Capacitación de } \\
\text { Innovación }\end{array}$} & $\begin{array}{c}\text { Coeficiente de } \\
\text { correlación }\end{array}$ & $1.000 * *$ & 1.000 \\
\hline & & Sig. (bilateral) & & \\
\hline & & $\mathrm{N}$ & 7 & 7 \\
\hline & $* *$. La & significativ & vel 0,01 (bila & \\
\hline
\end{tabular}

Fuente: Elaboración propia. 
Tabla 24. Análisis de correlación de Spearman para las subdimensiones Colaboración para Administrar y Financiamiento de Innovación en empresas del clúster

\begin{tabular}{|c|c|c|c|c|}
\hline \multicolumn{5}{|c|}{ Correlaciones en empresas del clúster entre subdimensiones } \\
\hline & & & $\begin{array}{c}\text { Colaborar para } \\
\text { Administrar }\end{array}$ & $\begin{array}{l}\text { Financiamiento de } \\
\text { Innovación }\end{array}$ \\
\hline \multirow{6}{*}{$\begin{array}{c}\text { Rho de } \\
\text { Spearman }\end{array}$} & \multirow{3}{*}{$\begin{array}{l}\text { Colaborar para } \\
\text { Administrar }\end{array}$} & Coeficiente de correlación & 1.000 & $1.000 * *$ \\
\hline & & Sig. (bilateral) & & \\
\hline & & $\mathrm{N}$ & 7 & 7 \\
\hline & \multirow{3}{*}{$\begin{array}{c}\text { Financiamiento } \\
\text { de Innovación }\end{array}$} & Coeficiente de correlación & $1.000 * *$ & 1.000 \\
\hline & & Sig. (bilateral) & & \\
\hline & & $\mathrm{N}$ & 7 & 7 \\
\hline
\end{tabular}

Fuente: Elaboración propia.

El análisis de correlación entre las dimensiones y subdimensiones significativas para las empresas encuestadas ajenas al clúster se muestran de la tabla 25 a la 29.

La tabla 25 muestra el análisis de correlación de Spearman entre las dimensiones de este trabajo, que incluyen a las actividades de colaboración e innovación, contemplando a los 7 encuestados de las empresas ajenas al clúster. Como resultado se alcanza una significancia aceptable menor al 0.05 y una correlación entre 0.790 a 1.000 . 
Tabla 25. Análisis de correlación de Spearman para las dimensiones en empresas ajenas al clúster

\begin{tabular}{|c|c|c|c|c|}
\hline \multicolumn{5}{|c|}{ Correlaciones Empresas Ajenas al Clúster } \\
\hline & & & $\begin{array}{l}\text { Actividades de } \\
\text { Colaboración }\end{array}$ & $\begin{array}{l}\text { Actividades de } \\
\text { Innovación }\end{array}$ \\
\hline \multirow{6}{*}{$\begin{array}{c}\text { Rho de } \\
\text { Spearman }\end{array}$} & \multirow{3}{*}{$\begin{array}{l}\text { Actividades de } \\
\text { Colaboración }\end{array}$} & Coeficiente de correlación & 1.000 & $.790^{*}$ \\
\hline & & Sig. (bilateral) & & 0.035 \\
\hline & & $\mathrm{N}$ & 7 & 7 \\
\hline & \multirow{3}{*}{$\begin{array}{l}\text { Actividades de } \\
\text { Innovación }\end{array}$} & Coeficiente de correlación & $.790^{*}$ & 1.000 \\
\hline & & Sig. (bilateral) & 0.035 & \\
\hline & & $\mathrm{N}$ & 7 & 7 \\
\hline
\end{tabular}

Fuente: Elaboración propia.

La tabla 26 muestra el análisis de correlación de Spearman entre la subdimensión de colaboración colaborar para producir y la subdimensión de agentes de innovación. Como resultado contemplando a las empresas ajenas al clúster, se alcanza una alta significancia aceptable al 0.05 y una correlación desde 0.762 hasta 1.000 . 
Tabla 26. Análisis de correlación de Spearman para las subdimensiones Colaboración para Producir y Agentes de Innovación en empresas ajenas al clúster

\begin{tabular}{|c|c|c|c|c|}
\hline \multicolumn{5}{|c|}{ Correlaciones en empresas del clúster entre subdimensiones } \\
\hline & & & $\begin{array}{l}\text { Colaborar para } \\
\text { Producir }\end{array}$ & $\begin{array}{l}\text { Agentes de } \\
\text { Innovación }\end{array}$ \\
\hline \multirow{6}{*}{$\begin{array}{c}\text { Rho de } \\
\text { Spearman }\end{array}$} & \multirow{3}{*}{$\begin{array}{l}\text { Colaborar para } \\
\text { Producir }\end{array}$} & $\begin{array}{c}\text { Coeficiente de } \\
\text { correlación }\end{array}$ & 1.000 & $.762 *$ \\
\hline & & Sig. (bilateral) & & 0.046 \\
\hline & & $\mathrm{N}$ & 7 & 7 \\
\hline & \multirow{3}{*}{ Agentes de Innovación } & $\begin{array}{c}\text { Coeficiente de } \\
\text { correlación }\end{array}$ & $.762 *$ & 1.000 \\
\hline & & Sig. (bilateral) & 0.046 & \\
\hline & & $\mathrm{N}$ & 7 & 7 \\
\hline
\end{tabular}

Fuente: Elaboración propia.

La tabla 27 muestra el análisis de correlación de Spearman entre la subdimensión de colaboración colaborar para innovar y la subdimensión de agentes de innovación. Como resultado contemplando a las empresas ajenas al clúster, se alcanza una alta significancia aceptable al 0.05 y una correlación desde 0.803 hasta 1.000 . 
Tabla 27. Análisis de correlación de Spearman para las subdimensiones Colaboración para Innovar y Agentes de Innovación en empresas ajenas al clúster

\begin{tabular}{|c|c|c|c|c|}
\hline \multicolumn{5}{|c|}{ Correlaciones en empresas ajenas al clúster entre subdimensiones } \\
\hline & & & $\begin{array}{l}\text { Colaborar para } \\
\text { Innovar }\end{array}$ & $\begin{array}{l}\text { Agentes de } \\
\text { Innovación }\end{array}$ \\
\hline \multirow{6}{*}{$\begin{array}{c}\text { Rho de } \\
\text { Spearman }\end{array}$} & \multirow{3}{*}{$\begin{array}{l}\text { Colaborar para } \\
\text { Innovar }\end{array}$} & Coeficiente de correlación & 1.000 & $.803^{*}$ \\
\hline & & Sig. (bilateral) & & 0.030 \\
\hline & & $\mathrm{N}$ & 7 & 7 \\
\hline & \multirow{3}{*}{$\begin{array}{l}\text { Agentes de } \\
\text { Innovación }\end{array}$} & Coeficiente de correlación & $.803 *$ & 1.000 \\
\hline & & Sig. (bilateral) & 0.030 & \\
\hline & & $\mathrm{N}$ & 7 & 7 \\
\hline \multicolumn{5}{|c|}{ **. La correlación es significativa en el nivel 0,01 (bilateral). } \\
\hline
\end{tabular}

Fuente: Elaboración propia.

La tabla 28 muestra el análisis de correlación de Spearman entre la subdimensión de colaboración colaborar para mercado y la subdimensión de agentes de innovación. Como resultado contemplando a las empresas ajenas al clúster, se alcanza una alta significancia aceptable al 0.05 y una correlación desde 0.803 hasta 1.000 . 
Tabla 28. Análisis de correlación de Spearman para las subdimensiones Colaboración para Mercado y Agentes de Innovación en empresas ajenas al clúster

\begin{tabular}{|c|c|c|c|c|}
\hline \multicolumn{5}{|c|}{ Correlaciones en empresas ajenas al clúster entre subdimensiones } \\
\hline & & & $\begin{array}{c}\text { Colaborar para } \\
\text { Mercado }\end{array}$ & $\begin{array}{l}\text { Agentes de } \\
\text { Innovación }\end{array}$ \\
\hline \multirow{6}{*}{$\begin{array}{c}\text { Rho de } \\
\text { Spearman }\end{array}$} & \multirow{3}{*}{$\begin{array}{c}\text { Colaborar para } \\
\text { Mercado }\end{array}$} & Coeficiente de correlación & 1.000 & $.803^{*}$ \\
\hline & & Sig. (bilateral) & & 0.030 \\
\hline & & $\mathrm{N}$ & 7 & 7 \\
\hline & \multirow{3}{*}{$\begin{array}{l}\text { Agentes de } \\
\text { Innovación }\end{array}$} & Coeficiente de correlación & $.803^{*}$ & 1.000 \\
\hline & & Sig. (bilateral) & 0.030 & \\
\hline & & $\mathrm{N}$ & 7 & 7 \\
\hline
\end{tabular}

Fuente: Elaboración propia.

La tabla 29 muestra el análisis de correlación de Spearman entre la subdimensión de colaboración colaborar para administrar y la subdimensión de agentes de innovación. Como resultado contemplando a las empresas ajenas al clúster, se alcanza una alta significancia aceptable al 0.05 y una correlación desde 0.803 hasta 1.000 . 
Tabla 29. Análisis de correlación de Spearman para las subdimensiones Colaboración para Administrar y Agentes de Innovación en empresas ajenas al clúster

\begin{tabular}{|c|c|c|c|c|}
\hline \multicolumn{5}{|c|}{ Correlaciones en empresas ajenas al clúster entre subdimensiones } \\
\hline & & & $\begin{array}{c}\text { Colaborar para } \\
\text { Administrar }\end{array}$ & $\begin{array}{l}\text { Agentes de } \\
\text { Innovación }\end{array}$ \\
\hline \multirow{6}{*}{$\begin{array}{c}\text { Rho de } \\
\text { Spearman }\end{array}$} & \multirow{3}{*}{$\begin{array}{c}\text { Colaborar para } \\
\text { Administrar }\end{array}$} & Coeficiente de correlación & 1.000 & $.762^{*}$ \\
\hline & & Sig. (bilateral) & & 0.046 \\
\hline & & $\mathrm{N}$ & 7 & 7 \\
\hline & \multirow{3}{*}{$\begin{array}{l}\text { Agentes de } \\
\text { Innovación }\end{array}$} & Coeficiente de correlación & $.762 *$ & 1.000 \\
\hline & & Sig. (bilateral) & 0.046 & \\
\hline & & $\mathrm{N}$ & 7 & 7 \\
\hline
\end{tabular}

Fuente: Elaboración propia.

\section{Análisis de resultados}

De acuerdo con los resultados presentados en cada una de las tablas, existen múltiples correlaciones de preguntas asociadas a colaboración con preguntas de innovación, éstas van de moderadas a altas correlaciones $\mathrm{y}$ todas se encuentran sustentadas con significancia suficiente de al menos al 0.05 y una confiablidad de aceptable a alta.

Lo anterior, sustenta el hecho de la existencia de una correlación positiva entre las variables de colaboración e innovación, para las empresas pertenecientes al clúster CELESA.

Por otro lado, en las empresas ajenas al clúster también se refleja una correlación positiva entre las variables de colaboración e innovación, sin embargo, únicamente se logra una significancia suficiente para concluirlo en una subdimensión de análisis, los agentes que producen innovación en las empresas.

\section{Conclusiones}

Este trabajo de investigación, está orientado al clúster CELESA como estudio de caso, teniendo como finalidad especificar la relación que existe entre la colaboración y la innovación entre las empresas que lo conforman.

Para establecer la correlación, se diseñó un instrumento que permite identificar elementos asociados a la colaboración e innovación en las empresas, una vez obtenidos los resultados con este formulario, se analizan 
en confiabilidad con Alfa de Cronbach y mediante un análisis de correlación bivariado de Spearman, se identifica la significatividad del resultado y el vínculo correlacional entre las variables.

Con base en los análisis de correlación bivariado de Spearman, presentados en la sección de resultados, a partir de cada pregunta en el instrumento diseñado y cuyos datos recolectados fueron verificados en confiabilidad mediante Alfa de Cronbach, y en validez mediante el resumen de procesamiento de casos. Se ha encontrado en este trabajo, que existe una relación significativamente positiva entre la colaboración y la innovación en empresas que pertenecen al clúster CELESA.

El análisis realizado en esta investigación, sustenta que en el clúster CELESA, el colaborar se ve gratificado con resultados que impulsan a las empresas en innovación, desde la generación de nuevos productos o servicios, la mejora de procesos administrativos, acceso a financiamientos, hasta en el impacto y mejora de procesos de distribución y mercadeo, además, dependiendo de las asociaciones esto favorece la capacitación del personal y el incremento de los expertos contratados.

Al identificar esta correlación positiva entre colaboración e innovación, las empresas del clúster tendrán un análisis sustentado que justifica el colaborar con otras empresas del clúster, para buscar innovar en el mercado de sus empresas.

Contemplando todo lo anterior, se alcanza el objetivo del trabajo de investigación de establecer el nivel y tipo de correlación entre las variables de colaboración e innovación para el clúster CELESA.

\section{Trabajo a futuro}

En el análisis de este estudio de caso, se abarca únicamente a empresas del clúster y un conjunto de empresas fuera de éste, que claramente mostraron una menor colaboración comparadas con aquellas incluidas en el clúster CELESA. Sin embargo, por la cantidad de empresas en el clúster sólo se pudo acceder a datos de 14 empresas, siete del clúster y siete fuera de éste, por tal motivo, el estudio aún no es representativo para su generalización hacia otros clústeres, contemplando lo anterior y los resultados obtenidos, el instrumento debe extenderse a otros clústeres en el estado de Aguascalientes y con los datos recabados, deben analizarse nuevamente a las variables de colaboración e innovación, buscando extender los resultados aquí presentados a cualquier empresa en un clúster en el estado.

Por otro lado, gracias a las preguntas con significatividad probada en el análisis aplicado en la sección de resultados, se pueden eliminar aquellas preguntas del instrumento que carecen de un valor significativo, de manera que se facilite el llenado del formulario, cuando sea replicado en otros marcos de referencia.

\section{Referencias:}

Aimacaña, N. L. y Lara, C. P. (2019). El Diamante Competitivo. Una propuesta para el cluster de las microempresas del sector de la confección de la parroquia Pasa en Tungurahua. Bolentín de Coyuntura, 1(22), 24-26.

https://doi.org/10.31164/bcoyu.22.2019.721 
Akoglu, H. (2018). User's guide to correlation coefficients. Turkish Journal of Emergency Medicine, 18(3), 91-93. https://doi.org/10.1016/j.tjem.2018.08.001

Antolín-López, R., Martínez-del-Río, J. y CéspedesLorente, J. (2013). Cooperación y competencia como antecedentes de la innovación de producto. ¿Aplican las empresas nuevas y establecidas una lógica diferenciada? Investigaciones Europeas de Direccion y Economia de la Empresa, 19(1), 53-62. https://doi.org/10.1016/j.iedee.2012.09.001

Arias, M. E. y Alarcón, S. (2019). Sistemas regionales de innovación agroalimentarios de Colombia: un análisis factorial y de clúster para la industria. Cuadernos de Desarrollo Rural, 16(84). https://doi.org/10.11144/Javeriana.cdr16-84.sria

Bateman, T. S. y Snell, S. (2009). Administración: Liderazgo y colaboración en un mundo competitivo.

Becerra, F., Serna, H. M. y Naranjo, J. C. (2013). Redes empresariales locales, investigación y desarrollo e innovación en la empresa. Cluster de herramientas de Caldas, Colombia. Estudios Gerenciales, 29(127), 247-257.

https://doi.org/10.1016/J.ESTGER.2013.05.013

Berumen, S. y Palacios, O. (2009). Competitividad, clusters e innovación. Redalyc, (March). https://doi.org/10.13140/RG.2.1.1209.0005

Briones, G. (2003). Métodos y técnicas de investigación para las ciencias sociales. Trillas.

Castañeda Jiménez, J. (2011). Metodología de la investigación. McGraw-Hill Interamericana.

Castro-Macías, J. Á. (2012). Relación entre Integración Empresarial y Competitividad en las Empresas que lo Conforman. Instituto Tecnológico de Aguascalientes.
Cuesta-Delgado, D. (2016). Surgimiento y evolución de clusters de empresas de base tecnológica en regiones de bajo desarrollo tecnológico : una mirada al caso de Cuatro Ríos Tecnologías, Cuenca, Ecuador. Pymes, Innovación y Desarrollo, 3(2-3).

Diaz-Ariza, D. M., García-Castiblanco, C. P., Pinzón Muñoz, C. A., Díaz-Ariza, D. M., García-Castiblanco, C. P. y Pinzón Muñoz, C. A. (2019). Programas gubernamentales para la internacionalización de las empresas: una reflexión desde el caso del sector de cosméticos y productos de aseo en Bogotá. Innovar, 29(71), 69-78.

https://doi.org/10.15446/innovar.v29n71.76396

Díaz-Carrión, I. A., Cruz-Estrada, I. y Páez-Pérez, Z. J. (2019). Entretejiendo innovación y colaboración en los Colectivos de Food Truck de Tijuana (México). Estudios Sociales. Revista de Alimentación Contemporánea y Desarrollo Regional, 29(53). https://doi.org/10.24836/es.v29i53.646

Domareski-Ruiz, T. C. y Chim-Miki, A. F. (2005). Competitividad e innovación: Teoría versus Práctica en la medida de competitividad turística. El periplo sustentable, (36), 134-156. Recuperado de http://www.scielo.org.mx/scielo.php?pid=S187090362019000100134\&script=sci_arttext\&tlng=pt

Fernández-Hurtado, R. S., Castillo-Triana, D. y Martínez-Martínez, L. Á. (2018). Clúster virtual nueva alternativa a la competitividad eficaz en las empresas. Tendencias, ISSN 0124-8693, ISSN-e 2539-0554, Vol. 19, $N^{o}$. 1, 2018 (Ejemplar dedicado a: Vol. XIX No. 1 Primer Semestre Enero - Junio 2018), págs. 164-186, 19(1), 164-186. Recuperado de https://dialnet.unirioja.es/servlet/articulo?codigo=6618 125 
Hernández-Sampieri, R., Fernández-Collado, C. y Baptista-Lucio, P. (2006). Metodología de la investigación. Recuperado de https://books.google.com.mx/books/about/Metodología _de_la_investigación.html?id=4_-

kPwAACAAJ\&redir_esc $=y$

IIzábal, E. y Valenzuela, B. (2016). Cooperar para Innovar, El caso de las iniciativas clúster del sector TI de Sinaloa, México. Desarrollo regional. Modelos, actores, gestión, 1(3), 37-52.

León, S. M. M. y Rodríguez, V. O. (2017). Estudio comparativo del desarrollo económico y cambio de especialización productiva de dos regiones en México María. Memoria del XI Congreso de la Red Internacional de Investigadores en Competitividad, 879-898.

Martínez, M.L. y Feria, C.M. (2016). Las PYMEs del clúster CRAA: un estudio de relación entre innovación y competitividad en Aguascalientes. En Gestión de la Innovación y la Tecnología (pp. 1-18). ACACIA.

Ojeda Goméz, J. (2009). La cooperación empresarial como estrategia de las PYMES del sector ambiental. Estudios gerenciales, 25(110), 39-61.

Pardo, A. y Ruiz, M. A. (2002). SPSS 11: Guía para el análisis de datos. Recuperado de https://bit.ly/2pvqT22

Peres, W. y Garrido, C. (1998). Grandes empresas y grupos industriales Latinoamericanos: expansión y desafíos en la era de la apertura y la globalización. Siglo vientiuno editores.
Reche, F. H. (2019). ¿¿Un clúster industrial en América Latina? La industria mexicana del calzado de cuero en León, Guanajuato. Observatorio de la economía latinoamericana. Recuperado de https://www.eumed.net/rev/oel/2019/06/industriamexicana-calzado.html

Rodríguez-Albor, G. (2018). Innovación y competitividad en la industria de software. Revista Venezolana de Gerencia, 23(83), 680-698. https://doi.org/10.31876/revista.v23i83.24497

Stevenson, W. J. (2005). Estadística para administración y economía : conceptos y aplicaciones. Alfaomega.

Tomer, A., Castillo-Navarro, A., Barcelo-Valenzuela, M. y León-Duarte, J. A. (2009). Importancia de los clusters del conocimiento como estrategia para gestionar el conocimiento entre organizaciones. Intangible Capital, 5(1). Recuperado de https://upcommons.upc.edu/handle/2099/7142

Zavala, A. G. (2014). IndustriaElectrónica. Recuperado de

https://www.gob.mx/cms/uploads/attachment/file/7633 9/141216_DS_Electronico_ES.pdf

Zayas, B.I., Zayas, B.R.A. y Heiras, P.M. de J. (2017). Clúster agricola como factor de desarrollo en las empresas del municipio de Angostura Sinaloa [Agricultural cluster development as a factor in companies in the municipality of Angostura Sinaloa]. Revista Mexicana de Agronegocios. 